Published online 2017 April 13.

Abstract

\title{
Assessment of Radio-Guided Occult Lesion Localization Associated with Sonography in Nonpalpable Breast Lesions
}

\author{
Ali Alamdaran, ${ }^{1, *}$ Ramin Sadegi, ${ }^{1}$ Aida Sharifi, ${ }^{1}$ Elaheh Moododi, ${ }^{1}$ and Naser Forghani ${ }^{1}$ \\ ${ }^{1}$ Mashhad University of Medical Sciences,Mashhad,Iran \\ "Corresponding author: Ali Alamdaran. E-mail: alamdarana@mums.ac.ir
}

Received 2016 December 21; Accepted 2017 February 08.

\begin{abstract}
Background: Several methods are in use for localization of non-palpable breast lesions prior to excision. The most common is wire-guided localization which is fraught with difficulties and possible complications. Radio-guided localization of non-palpable lesions (ROLL) is a relatively new method which has been never used in our country before. We have for the first time investigated its diagnostic value for localization of non-palpable breast lesions in a teaching hospital in Iran.

Methods: In this study, 42 patients with non-palpable breast lesions, detected by ultrasonography, underwent radio-guided localization. Phytate-Tc-99m was injected into the lesion under the guidance of ultrasonography, 1-2 hours before surgery. Excision of the lesion was performed according to radioactivity signal of lesion, detected by a hand-held gamma probe during surgery. After total excision, the resection bed was explored by gamma probe to detect any residual activity. So if there was no radioactivity signal, the excision was regarded as complete. The data of ultrasonographic characteristics, localization and excision procedure, complications, margin status and tissue volume were analyzed by SPSS 16.

Results: Pathologic results were benign in $90.5 \%$ and malignant in $9.5 \%$ of patients. Post-operative margin of lesion was clear in $100 \%$ of patients. No margin involvement was reported. Mean duration of localization and surgical procedure was $1.80 \pm 0.42$ and $26.78 \pm 8.10$ minutes, respectively. Fault in localization took place in 4 patients (9.5\%) and there was a need to secondary surgery in 1 patient (2.4\%). Patients' satisfaction level about post-operative cosmetic results was excellent in $33.3 \%$ and good in $66.6 \%$ of patients. Mean excised tissue volume was $28.59 \pm 27.96 \mathrm{~cm}$ in all patients and $23.54 \pm 23.46 \mathrm{~cm}$, in patients without fault of localization.

Conclusions: Radio-guided localization of non-palpable breast lesions is an acceptable method because of technical facilitation, accurate localization and complete excision with limited complications. So it can be recommended as an alternative method for wire-guided localization.
\end{abstract}

This is an abstract presented in the 33rd Iranian congress of radiology (ICR) and the 15th congress of Iranian radiographic science association (IRSA). 